\title{
Caracterização de mini-implantes utilizados na ancoragem ortodôntica
}

Luciana Rougemont Squeff*, Michel Bernard de Araújo Simonson**,

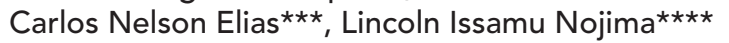

\section{Resumo}

Introdução: o diâmetro reduzido do mini-implante, e a decorrente facilidade na sua inserção, minimizam a possibilidade de erro do operador e de contato entre a rosca do mini-implante e a raiz dentária. Entretanto, o risco de fratura da peça aumenta à medida que seu diâmetro é diminuído. Métodos: neste trabalho foram analisados quatro produtos de marcas nacionais (INP, SIN, Conexão e Neodente) e um de marca alemã (Mondeal), com o objetivo de identificar características importantes para o bom desempenho deste recurso como acessório de ancoragem. Foram observados composição e design das peças e realizado o ensaio mecânico de torque até a fratura (estudo in vitro), cujos valores foram submetidos à análise de variância (ANOVA) e teste de Tukey. Resultados: os resultados mostraram que todos os mini-implantes testados estão aptos à utilização clínica como reforço de ancoragem ortodôntica.

Palavras-chave: Mini-implante. Ancoragem esquelética.

\section{INTRODUÇÃO}

Sistemas de ancoragem esquelética têm sido amplamente difundidos e utilizados em Ortodontia, pois possibilitam resultados satisfatórios no controle da ancoragem, com menor incômodo para o paciente. Uma vez que estes dispositivos podem substituir a utilização de recursos extra e intrabucais, que dependem mais da colaboração do paciente, a perda de ancoragem pode ser facilmente evitada ${ }^{5,7,13,14}$.

Os mini-implantes apresentam-se como uma técnica simples e pouco invasiva, sem necessidade da utilização de terapia medicamentosa antes ou após sua inserção, sendo confortáveis para o paciente. Tal alternativa de ancoragem é bastante recomendada para resolver problemas complexos em Ortodontia ${ }^{1,22}$, podendo ser indicada em casos onde o paciente apresenta-se com número insuficiente de dentes para a aplicação de recursos convencionais (cujas forças empregadas podem gerar efeitos reativos adversos), sendo necessária a movimentação dentária assimétrica em todos os planos do espaço e, algumas vezes, como alternativa à cirurgia ortognática ${ }^{10}$. Os sistemas utilizados originaram-se de duas linhas diferentes. $\mathrm{O}$ primeiro tipo, proveniente do implante dentário

* Doutoranda em Odontologia (Ortodontia) - UFRJ. Mestre em Odontologia (Ortodontia) - UFRJ.

** Tecnólogo do laboratório de Metalografia/ Microscopia de CEPEL. Tecnólogo em Telecomunicações (Faculdade Estácio de Sá). Técnico em MecâTecnólogo do labo

*** Professor do Laboratório de Biominerais do Instituto Militar de Engenharia/RJS.

**** Professor Adjunto do Programa de Pós-Graduação em Odontologia (Ortodontia) - UFRJ. 
osteointegrado, o qual tem base científica sólida de estudos clínicos ${ }^{1,5,8}$, biomecânicos ${ }^{3,4}$ e histológicos ${ }^{19}$. Dentre estes estão os implantes ortodônticos, os quais são de menor tamanho que os implantes dentários convencionais, mas com superfícies tratadas de maneira semelhante, promovendo osteointegração. Incluem-se nesta categoria os implantes retromolares e os palatinos. Ambos são utilizados para ancoragem indireta, uma vez que estão conectados aos dentes que funcionam como unidades de ancoragem. O segundo tipo de sistema originou-se dos mini-implantes, tendo sido especificamente projetados para uso ortodôntico, como ancoragem direta. Em seguida, foi criado um novo tipo de implante com extremidade semelhante a um braquete, que pode ser utilizado para ancoragem direta e indireta. Diferentes dos implantes osteointegrados, estes possuem diâmetro menor, têm superfícies lisas e são projetados para aplicação de força imediata ou pouco tempo após sua inserção ${ }^{10}$.

Implantes promovem pontos de ancoragem efetiva para movimentações dentárias, mesmo quando é realizada carga imediata ${ }^{11}$. A utilização deste recurso para casos onde se faz necessária a intrusão de molares tem-se mostrado bastante apropriada, uma vez que promove vetores verticais de força, sem a presença de forças extrusivas recíprocas nos outros dentes ${ }^{6,9}$. Além disso, a utilização de aparelhos extrabucais ou barras transpalatinas, bem como a inclusão dos segundos molares para reforço na ancoragem, pode ser evitada quando utilizado este tipo de acessório, além da indicação para casos onde é necessário o tracionamento de caninos impactados ${ }^{6,10}$ ou verticalização de molares ${ }^{16}$.

Os mini-implantes são, em sua maioria, confeccionados de liga de titânio. Variam quanto à forma, design e medidas, de acordo com a marca comercial. Possuem três porções distintas: cabeça - área para instalação de dispositivos ortodônticos; porção transmucosa ou pescoço - região existente entre a porção rosqueável e a cabeça do implante (geralmente lisa, acomoda os tecidos peri-implantares) e porção rosqueável - parte ativa do miniimplante ${ }^{14}$.

A cabeça do mini-implante pode ter um orifício, um gancho ou botão em sua extremidade. Também pode ser encontrada com design de braquete, oferecendo a vantagem de proporcionar o controle tridimensional, bem como a ancoragem indireta. Nesta porção, são acoplados dispositivos tais como molas, elásticos ou fios de amarrilho, para ancoragem ou movimentação, conforme o planejamento ${ }^{13,14}$.

A porção transmucosa deve apresentar-se de comprimentos variados, possibilitando sua colocação em diferentes sítios ${ }^{6}$. Outra característica importante a ser observada nesta porção do miniimplante é o polimento. Quanto mais bem polida apresentar-se esta parte da peça menor a possibilidade de infecção nos tecidos adjacentes ${ }^{10}$.

A porção rosqueável varia nos diâmetros de 1 a $2 \mathrm{~mm}$, sendo o corte da rosca característica importante a ser considerada na escolha da peça ${ }^{10}$. Os mini-implantes perfurantes têm o ápice extremamente fino e afiado, dispensando, na maioria dos casos, a utilização de qualquer procedimento adicional para perfuração óssea, enquanto os de ápice arredondado necessitam de perfuração com broca, no sítio onde serão posicionados, sendo estes denominados implantes auto-rosqueantes ${ }^{20}$.

O diâmetro do mini-implante deve ser escolhido de acordo com o sítio e o espaço disponível no mesmo, através de radiografia intrabucal. $\mathrm{Na}$ maxila, deve-se selecionar uma peça de diâmetro mais fino, quando a mesma for colocada entre as raízes de dentes. Se for necessária a inserção do implante em osso trabecular, para obtenção de estabilidade, uma peça de comprimento mais longo se faz necessária. Entretanto, se o osso cortical já for suficiente para torná-lo estável, uma peça de comprimento menor pode ser selecionada. Sítios de inserção possíveis na maxila são: a área abaixo da espinha nasal, o palato, o processo alveolar, a crista infra-zigomática, devendo o mini-implante 
ser colocado em ângulo oblíquo e em direção apical. Na mandíbula, as áreas de escolha para a colocação do mini-implante são o processo alveolar, a área retromolar e a sínfise mandibular, devendo a inserção ser feita paralela às raízes, quando na presença de dentes. O mini-implante transcortical pode ser utilizado para dar mais estabilidade à área edêntula, onde o osso trabeculado é geralmente escasso ${ }^{10}$. A vantagem de utilizar mini-implantes que possuem menor diâmetro é a facilidade de inserção entre as raízes, diminuindo o risco de contato radicular. Alguns problemas são relatados quando da utilização de mini-implantes ${ }^{2}$. Dentre eles, um dos mais comuns é a ocorrência de fratura ${ }^{3}$. O risco de fratura está intimamente relacionado ao diâmetro do implante utilizado, uma vez que, geralmente, ocorre em casos de implantes de diâmetro muito fino ou cujo pescoço não seja resistente o suficiente para suportar a tensão no momento da sua remoção ${ }^{3}$. Para evitar tal incidente, deve-se utilizar peças cônicas de diâmetro apropriado para a qualidade do sítio ósseo escolhido. A fratura também pode ocorrer por falha do operador, em caso de aplicação de força excessiva na colocação de mini-implante do tipo auto-rosqueável ou autoperfurante. Outro problema comum advém da utilização de miniimplantes com porção transmucosa mal polida, o que predispõe à infecção nos tecidos locais ${ }^{10}$. A higiene bucal pós-cirúrgica é outro fator de suma importância para a estabilidade do mini-implante, sendo imprescindível orientar o paciente quanto às medidas necessárias para controle do biofilme, bem como realizar consultas semanais para controle clínico no primeiro mês ${ }^{13}$.

O objetivo da presente pesquisa é caracterizar, num estudo in vitro, mini-implantes de ancoragem ortodôntica de cinco diferentes fabricantes (SIN, INP, Conexão, Neodent e Mondeal), em relação à topografia, ensaio mecânico de torque até a fratura e design da peça, apresentando dados que auxiliem na produção e qualidade de miniimplantes nacionais, o que irá promover o maior
Tabela 1 - Distribuição da amostra analisada.

\begin{tabular}{ccccc} 
marca & $\begin{array}{c}\text { diâmetro } \\
(\mathrm{mm})\end{array}$ & $\begin{array}{c}\text { comprimento } \\
(\mathrm{mm})\end{array}$ & liga & sistema \\
\hline SIN & 1,4 & 8,0 & Ti-6AL-4V & autoperfurante \\
SIN & 1,6 & 8,0 & Ti-6AL-4V & autoperfurante \\
\hline INP & 1,5 & 8,0 & Ti-6AL-4V & autorosqueante \\
\hline Conexão & 1,5 & 8,0 & Ti-6AL-4V & autoperfurante \\
\hline Neodente & 1,6 & 7,0 & Ti-6AL-4V & autoperfurante \\
\hline Mondeal & 1,5 & 7,0 & Ti-6AL-4V & autoperfurante \\
\hline
\end{tabular}

aproveitamento dos mesmos na utilização como reforço de ancoragem ortodôntica.

\section{MATERIAL E MÉTODOS}

Foram analisados 30 mini-implantes (5 peças de cada grupo), utilizados como reforço de ancoragem ortodôntica, com as especificações citadas na tabela 1 .

\section{Microscopia eletrônica de varredura (MEV) Fotomicrografia}

Para observação da topografia e design das peças, as mesmas foram montadas em bases de alumínio próprias para este fim, com fita dupla face de carbono e levadas ao microscópio eletrônico de varredura. $\mathrm{O}$ equipamento utilizado foi o modelo LEO 940 (Zeiss, Alemanha), na faixa de alto vácuo, com $20 \mathrm{kv}$ de aceleração e corrente do filamento de $0,8 \mu \mathrm{A}$. Foram realizadas fotomicrografias de $25 \mathrm{x}$, 50x, 100x e 200x dos mini-implantes estudados, sendo obtidas imagens da cabeça, da porção transmucosa e da porção rosqueável das peças.

\section{Análise por dispersão de RX (EDX)}

A liga metálica dos mini-implantes foi caracterizada por dispersão de raios-X, no microscópio eletrônico de varredura. Para isso, as peças foram cortadas em Isomet (Buehler, Illinois, USA) e lavadas em equipamento de ultra-som Ultramet 2002 (Buehler, Illinois, USA). Em seguida, foram cuidadosamente secas e posicionadas nas bases próprias para MEV, para análise da composição. 


\section{Medições em projetor de perfil digital}

O projetor de perfil digital (Fig. 1) Pantec (Panambra Industrial e Técnica S.A., São Paulo, Brasil) foi utilizado para obtenção de duas medidas importantes para a avaliação do design das peças: foram avaliadas a profundidade das roscas e a distância inter-roscas.

\section{Ensaio mecânico de torque}

Os mini-implantes foram submetidos ao ensaio mecânico de torque ${ }^{4}$, sendo cada peça inserida em cortical de tíbia suína, até que ocorresse fratura. Inicialmente, a tíbia suína foi fixada em torno de bancada, para que não sofresse nenhum movimento durante a inserção dos mini-implantes. Realizou-se a confecção do orifício guia com fresa cirúrgica de 1,0mm de diâmetro. Em seguida, a chave manual própria de cada sistema de mini-implantes foi fixada no cabeçote do torquímetro digital (Lutron TQ - 8800, Taiwan). A inserção dos mini-implantes foi executada pelo mesmo operador até a fratura, sendo realizados ensaios de cinco peças de cada tipo de mini-implante. Os valores de torque de inserção obtidos foram submetidos à análise de variância (ANOVA) e teste de Tukey, bem como à análise estatística descritiva (Tab. 4).

\section{RESULTADOS}

A figura 2 mostra o design dos mini-implantes estudados, com fotomicrografias obtida em MEV, podendo-se observar as regiões da cabeça e as porções rosqueáveis nas figuras 3 e 4 , respectivamente, em maior magnificação.

A tabela 2 mostra o percentual dos elementos químicos alumínio (Al) e titânio (Ti) encontrados nas peças estudadas, através da análise por dispersão de raios-X (EDX).

As médias das distâncias inter-roscas e as médias das profundidades das roscas dos mini-implantes estudados são apresentadas na tabela 3 .

$\mathrm{Na}$ tabela 4 , observa-se os valores mínimo e máximo das forças de torque de inserção, a média das forças empregadas até a ocorrência de fratura

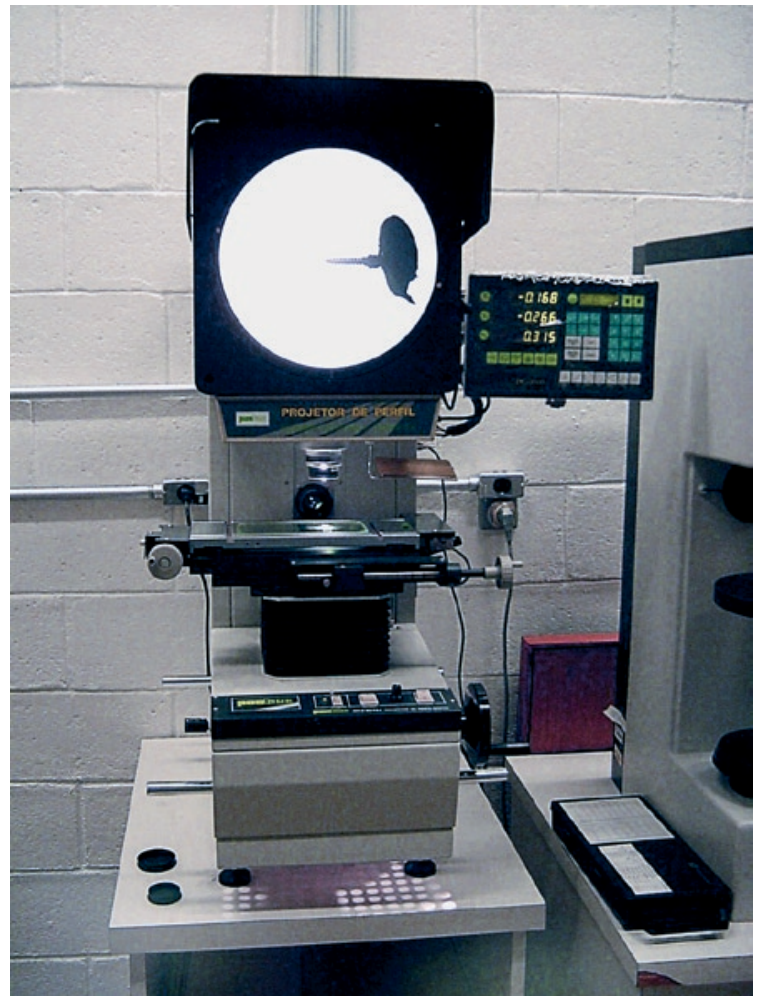

FIGURA 1 - Projetor de perfil Pantec.

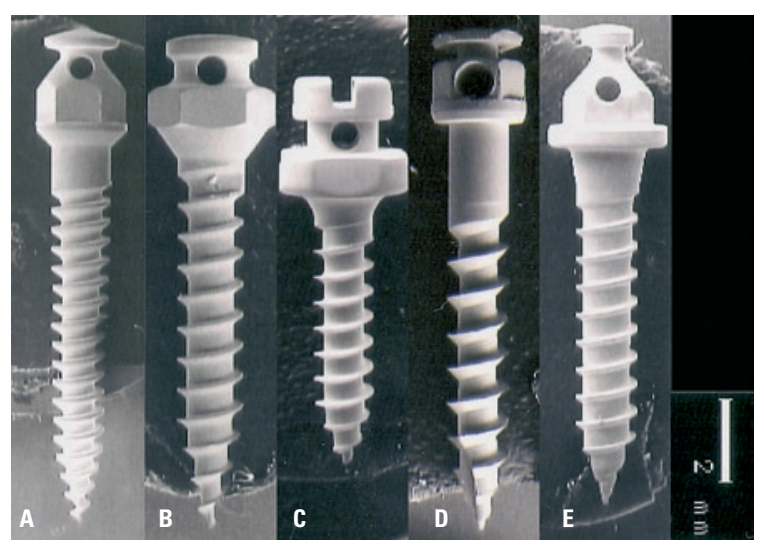

FIGURA 2 - Fotomicrografias em magnificância de 6x mostrando o design dos mini-implantes estudados: A) Conexão, B) Neodente, C) Mondeal, D) INP e E) SIN.

Tabela 2 - Elementos que compõem os sistemas de implantes estudados.

\begin{tabular}{|cccccc|}
\hline & SIN & INP & Conexão & Neodente & Mondeal \\
\hline Al (\%) & 2,60 & 2,60 & 2,51 & 2,18 & 2,50 \\
Ti (\%) & 97,40 & 97,40 & 97,49 & 97,82 & 97,50 \\
\hline
\end{tabular}




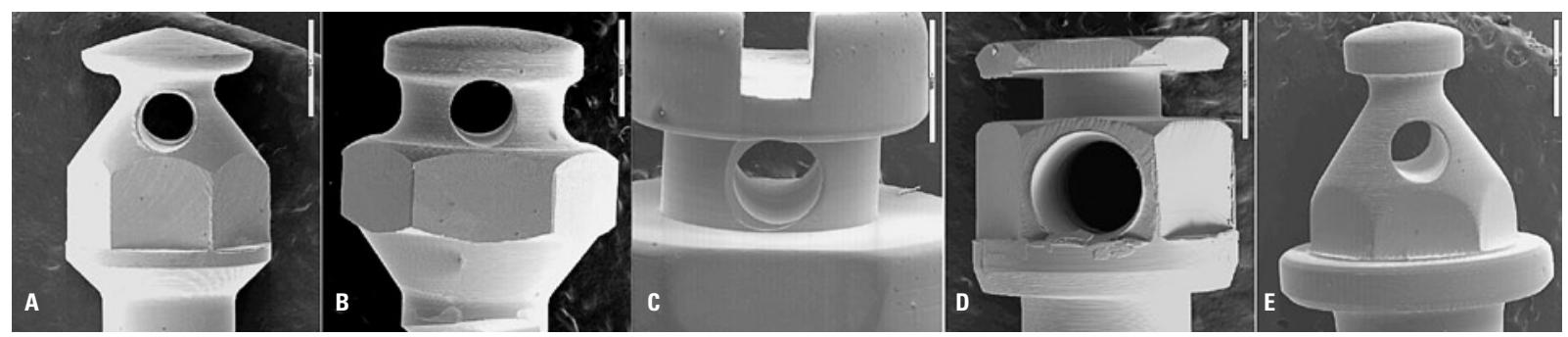

FIGURA 3 - Fotomicrografia da região da cabeça dos mini-implantes das marcas: A) Conexão, B) Neodente, C) Mondeal, D) INP e E) SIN (magnificação de 27x).

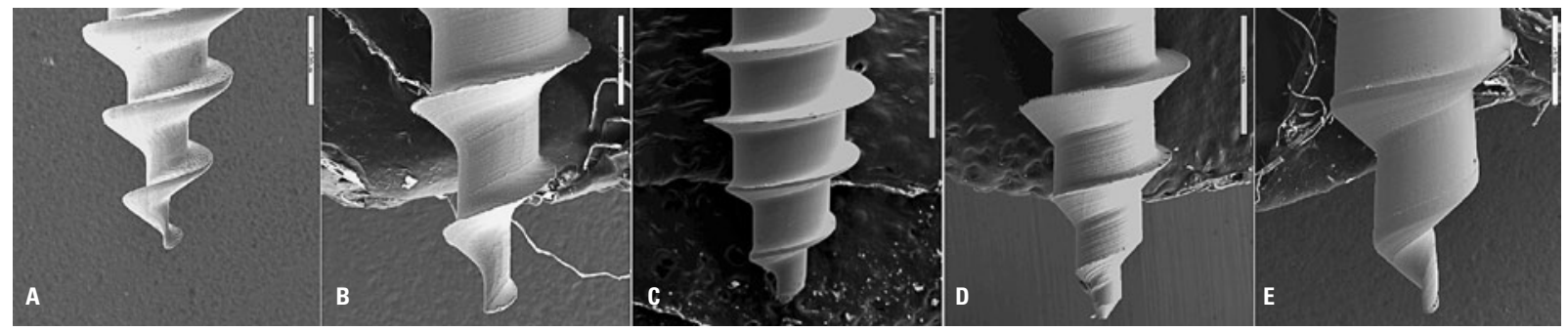

FIGURA 4 - Fotomicrografia da região das roscas dos mini-implantes das marcas: A) Conexão, B) Neodente, C) Mondeal, D) INP e E) SIN (magnificação de 50x).

Tabela 3 - Média da distâncias inter-roscas e profundidades das roscas dos mini-implantes estudados (mm).

\begin{tabular}{ccc}
$\begin{array}{c}\text { mini-implantes } \\
\text { estudados diâmetro } \\
\text { x comprimento }(\mathrm{mm})\end{array}$ & $\begin{array}{c}\text { média das distân- } \\
\text { cias inter-roscas } \\
(\mathrm{mm})\end{array}$ & $\begin{array}{c}\text { média das profun- } \\
\text { didades das roscas } \\
(\mathrm{mm})\end{array}$ \\
\hline SIN $1,4 \times 8,0$ & 0,796 & 0,186 \\
$\operatorname{SIN} 1,6 \times 8,0$ & 0,693 & 0,199 \\
INP $1,5 \times 8,0$ & 0,857 & 0,304 \\
\hline CONEXÃ $1,5 \times 8,0$ & 0,498 & 0,255 \\
\hline NEODENTE $1,6 \times 7,0$ & 0,734 & 0,243 \\
\hline MONDEAL $1,5 \times 7,0$ & 0,654 & 0,267 \\
\hline
\end{tabular}

das peças, o desvio-padrão e a estatística dos grupos de mini-implantes estudados.

\section{DISCUSSÃO}

Os mini-implantes ortodônticos são fabricados com a liga Ti-6AL-4V, diferente dos implantes dentários osteointegráveis, que são, geralmente, fabricados com titânio comercialmente puro. Isso se dá, basicamente, pelos seguintes motivos: os mini-implantes são menores em diâmetro que os
Tabela 4 - Análise estatística descritiva dos valores de torque de inserção obtidos nos grupos de mini-implantes estudados $\left(\mathrm{N} / \mathrm{cm}^{2}\right)$.

\begin{tabular}{|cccccc|} 
grupos & média & d.p. & mínimo & máximo & estatística \\
SIN $1,4 \times 8,0$ & 26,34 & 3,05 & 23,1 & 30,5 & AC \\
SIN $1,6 \times 8,0$ & 40,0 & 1,19 & 38,5 & 41,4 & D \\
INP $1,5 \times 8,0$ & 22,3 & 1,99 & 20,2 & 24,6 & AB \\
CON 1,5 88,0 & 18,26 & 1,06 & 17,4 & 20,0 & B \\
NEO 1,6 x 7,0 & 34,8 & 2,35 & 32,3 & 38,2 & E \\
MON 1,5 x 7,0 & 28,1 & 3,38 & 24,1 & 32,9 & C \\
\hline
\end{tabular}

Letras diferentes $=$ diferença estatisticamente significativa $(p>0,01)$.

implantes convencionais, sendo assim, faz-se necessária a utilização de material de maior resistência mecânica que o titânio comercialmente puro, como é o caso da liga Ti-6AL-4V. Esta liga possui características inferiores ao titânio comercialmente puro, em relação à bioatividade, o que faz com que a qualidade de osteointegração seja menor e a facilidade de remoção maior; além disso, os sistemas de mini-implantes baseiam-se na estabilidade mecânica primária (inicial), e não na estabi- 
lidade secundária, advinda da osteointegração $0^{3,18,19}$. Foi observada, em todas as marcas pesquisadas, composição bastante semelhante (Tab. 2), sendo as peças compostas em sua maior parte, por titânio e uma pequena porção de alumínio. $\mathrm{O}$ vanádio não foi observado no gráfico, por se apresentar em concentração abaixo da mínima detectável pelo EDX.

Em relação à eficiência clínica dos mini-implantes, são preocupantes as falhas relacionadas à sua utilização e possíveis causas de problemas como a peri-implantite e a fratura da peça. Um dos motivos de perda do mini-implante é o acúmulo de biofilme ao redor do implante ou agressão mecânica persistente, podendo causar problemas tais como inflamação aguda ou crônica e infecção ${ }^{17}$. Para evitar tais adversidades, alguns cuidados relacionados ao design dos miniimplantes devem ser tomados. É indicada a existência de pescoço transmucoso cilíndrico, para facilitar a união da peça ao tecido mole (interface implante-tecido mole) e a higienização. A porção transmucosa dos mini-implantes deve ser bem polida, para que se evite o acúmulo de biofilme nos tecidos locais ${ }^{10}$. Neste estudo, observou-se, através das fotomicrografias, que todas as marcas atendem a esse cuidado no polimento dos miniimplantes, tanto em regiões do pescoço quanto na cabeça das peças.

Outro cuidado importante no design é o diâmetro da cabeça do mini-implante, que deve ser mais largo que o pescoço transmucoso, para prevenir a cobertura da peça por tecido mole ${ }^{8}$. No presente trabalho, todos os implantes estudados apresentaram tal característica satisfatória. Outro ponto desejado em relação aos mini-implantes deve ser a sua facilidade e diversidade de aplicações. É bastante interessante a possibilidade de utilização de um mesmo mini-implante para aplicação direta e indireta, ou seja, a estrutura da cabeça deve ser anatomicamente desenhada para possibilitar o uso concomitante de elástico e de fio ortodôntico. Dos mini-implantes estudados, apenas o Mondeal apresentou slot que possibilita a colocação de fio ortodôntico (Fig. 3). As demais peças apresentam apenas botão e orifício para colocação de elásticos e molas.

O design deve assegurar à peça a prevenção contra danos tissulares irreversíveis, tais como injúria à raiz. Para isso, a porção apical da rosca deve ser mais estreita e o sistema de perfuração indicado bastante seguro, de forma a eliminar qualquer possibilidade de ocorrência de injúria permanente às estruturas anatômicas. Esta característica também facilita a inserção do mini-implante e minimiza o trauma cirúrgico. Nesta pesquisa, todos os mini-implantes estudados apresentaram a porção apical delgada (Fig. 4). A estabilidade primária é um pré-requisito para a cicatrização e está intimamente relacionada ao suporte ósseo. A rosca em forma de cone assegura o efeito de condensação do osso, melhorando sua qualidade e prevenindo destruição indesejável da cortical óssea, causada por inserção excêntrica ou mudança do eixo durante a inserção, o que faz com que a estabilidade do implante não seja muito influenciada pela habilidade do operador ou pelo local de inserção do implante $^{8}$. Das marcas estudadas, apenas as marcas Mondeal, INP e Conexão não apresentaram formato cônico na rosca, sendo observada forma cilíndrica na porção rosqueável das peças (Fig. 2).

Quanto à utilização de mini-implantes autoperfurantes ou auto-rosqueantes (sem e com procedimento de perfuração prévia, respectivamente), há controvérsias. Alguns autores acreditam que os mini-implantes autoperfurantes são os mais traumáticos, uma vez que este procedimento produz pressão física e microfraturas na região óssea adjacente, podendo ocorrer lesão no periósteo e no endósteo e necrose nas células ósseas. Entretanto, outros profissionais acreditam que o sistema de mini-implante auto-rosqueante causa maior trauma ósseo, devido ao calor friccional produzido pela fresa no ato de perfuração prévia. Há, ainda, os que preferem realizar a perfuração prévia com instrumento manual de alto poder cortante, para minimizar a produção de calor, realizando resfriamento com irrigação intensa, 
especialmente onde o osso é mais espesso ${ }^{8}$. Não foi objetivo deste estudo o teste in vivo, para concluir qual a melhor opção a ser utilizada. Porém, estudos clínicos mostram mais sucesso na utilização dos mini-implantes autoperfurantes, uma vez que estes apresentam maior estabilidade primária, quando comparados aos auto-rosqueantes ${ }^{15}$.

Os mini-implantes estudados apresentam comprimentos de $7 \mathrm{~mm}$ (Neodente e Mondeal) e $8 \mathrm{~mm}$ (SIN, INP e Conexão). Segundo Lee et al. ${ }^{8}$, o comprimento exerce pequeno efeito na distribuição da tensão, sendo o design da rosca e o diâmetro do mini-implante mais significativos em relação a esta característica. Os autores afirmam ser necessária a inserção de, no mínimo, $5 \mathrm{~mm}$ de comprimento do mini-implante no osso, entretanto, o aumento do comprimento da porção inserida, além desta medida, não significa aumento efetivo na estabilidade primária, a menos que se pretenda obter ancoragem bicortical. Os mini-implantes observados, portanto, estão de acordo com as indicações destes autores.

Em relação ao torque de inserção até a ocorrência de fratura dos mini-implantes estudados, os sistemas que tiveram a maior média de resistência à fratura por inserção foram os de maior diâmetro: $1,6 \mathrm{~mm}$, sendo eles os mini-implantes das marcas SIN (de 1,6mm) e Neodente (Tab. 4). Nossos achados estão de acordo com Elias, Guimarães e Muller ${ }^{3}$, que concluíram que quanto menor o diâmetro mais próximos os valores de força necessária para a inserção do mini-implante no osso e força de inserção necessária para fraturá-lo. Esses autores atribuem tal característica aos seguintes fatores: o torque é proporcional à área de contato do mini-implante com o osso, por ser utilizada - para preparação do alvéolo broca de menor diâmetro que o mini-implante, uma parte do torque se destina a cortar e alargar o orifício, quanto maior o diâmetro maior o volume de material a ser cortado e, durante a perfuração, restos de material ficam no interior do alvéolo, dificultando a rotação do mini-implante no momento da sua inserção. Isso nos mostra a necessidade de maior cuidado na utilização de mini-implantes de menor diâmetro, uma vez que a possibilidade de fratura aumenta. O terceiro melhor resultado foi apresentado pelo sistema Mondeal, cujo diâmetro é de $1,5 \mathrm{~mm}$. O mini-implante do Sistema SIN de 1,4mm de diâmetro apresentou o quarto melhor resultado, apesar do diâmetro menor em relação aos sistemas INP e Conexão. Não foi objetivo deste trabalho comparar as diferentes marcas de mini-implantes estudadas. Entretanto, observou-se haver diferença estatisticamente significativa entre alguns grupos de miniimplantes, como mostra a tabela 4. Cabe também ressaltar que a força de torque de inserção recomendada na clínica ortodôntica, segundo Motoyoshi et al. ${ }^{12}$, é de 5 a $10 \mathrm{~N} / \mathrm{cm}^{2}$, podendo chegar a $15 \mathrm{~N} / \mathrm{cm}^{2}$. Portanto, todos os implantes estudados apresentaram resultados satisfatórios em relação à resistência à fratura por inserção.

Todos os sistemas estudados sofreram fratura na porção ativa da rosca. As medições das distâncias inter-roscas e profundidades das roscas dos mini-implantes das cinco marcas foram realizadas (Tab. 3) e comparadas aos resultados obtidos no ensaio mecânico. Essas características se mostraram irrelevantes, não havendo associação entre a fragilidade do material na região da rosca (local da fratura) e tais medidas. Atenta-se para a necessidade de realização de maior número de estudos para que este problema seja identificado e solucionado. A existência de ranhura lateral na porção cortical da rosca do mini-implante confere maior resistência deste à fratura, pois previne a concentração excessiva de tensão nos tecidos adjacentes ao mini-implante, na área onde a rosca apresenta diâmetro mais ampliado ${ }^{8}$. Nenhum dos mini-implantes estudados apresentou tal modificação.

\section{CONCLUSÃO}

Após caracterização da topografia e design dos mini-implantes estudados, bem como do teste de torque, pode-se concluir que todos os miniimplantes testados estão aptos à utilização clínica como reforço de ancoragem ortodôntica. 


\section{AGRADECIMENTOS}

Os autores agradecem aos fabricantes dos sistemas INP, Mondeal, Neodente e Conexão pelo fornecimento dos mini-implantes. Ao Centro de Pesquisas de Energia Elétrica (CEPEL) e profissionais envolvidos, nosso agradecimento pelo apoio na utilização dos equipamentos de MEV e projetor de perfil digital. E à FAPERJ (Fundação Carlos Chagas Filho de Amparo à Pesquisa do Estado do Rio de Janeiro) pelo apoio financeiro.

\title{
Characterization of the mini-implants used to orthodontic anchorage
}

\begin{abstract}
Introduction: The reduced diameter of the mini-implants and the simplicity of the technique could minimize the operator mistake's possibility and avoid the contact between the thread of the screw and the dental root. In spite of that, it increases the fracture risk because of the diameter's decrease. Methods: At the present work, mini-implants from five different trade marks, four Brazilian systems (INP, SIN, Conexão and Neodente) and a German system (Mondeal) were studied to identify important characteristics of this anchorage accessory, like composition, design and resistance to the insertion torque (in vitro). The values obtained from the mechanical torque test were submitted to variance analysis (ANOVA) and Tukey's test. Results: All studied groups presented good results and are recommended to orthodontic clinic use.
\end{abstract}

Key words: Mini-implant. Skeletal anchorage.

\section{REFERÊNCIAS}

1. CARANO, A. et al. Clinical applications of the miniscrew anchorage system. J. Clin. Orthod., Boulder, v. 39, p. 9-24, Jan 2005.

2. CHENG, S. T. et al. A prospective study of the risk factors associated with failure of mini-implants used for orthodontic anchorage. Int. J. Oral Maxillofac. Implants, Copenhagen, v. 19, p. 100-106, 2004.

3. ELIAS, C. N.; GUIMARÃES, G. S.; MULLER, C. A. Torque de inserção e de remoção de mini-parafusos ortodônticos. RBI, Rio de Janeiro, v. 11, n. 3, p. 5-8, 2005.

4. ELIAS, C. N.; LOPES, H. P. Materiais dentários: ensaios mecânicos. São Paulo: Ed. Santos, 2007.

5. KANOMI, R. Mini-implant for orthodontic anchorage. J. Clin. Orthod., Boulder, v. 31, p. 763-767, Nov. 1997.

6. KESLING, P. Questions about miniscrews. J. Clin. Orthod., Boulder, v. 39, no. 9, p. 527-528, Sept. 2005.

7. KYUNG, S. H.; HONG, S. G.; PARK, Y. C. Distalization of maxillary molars with a midpalatal miniscrews. J. Clin. Orthod., Boulder, v. 37, no. 1, p. 22-25, Jan. 2003.

8. LEE, J. S. et al. Application of orthodontic mini-implants. 1st ed. Canadá: Quintessence, 2007.

9. MAINO, B. The spider screw for skeletal anchorage. J. Clin. Orthod., Boulder, v. 37, no. 2, p. 90-97, Feb. 2003.

10. MELSEN, B. Mini-implants, where are we? J. Clin. Orthod., Boulder, v. 39, no. 9, p. 539- 547, Sept. 2005.

11. MELSEN, B.; COSTA, A. Immediate loading of implants used for orthodontic anchorage. Clin. Orthod. Res., Copenhagen, v. 3, no. 1, p. 23-28, Feb. 2000.

12. MOTOYOSHI, M. et al. Recommended placement torque when tightening an orthodontic mini-implant. Clin. Oral Implants Res., Copenhagen, v. 17, no. 1, p. 109-114, Feb. 2006.

13. NASCIMENTO, M. H. A.; ARAÚJO, T. M.; BEZERRA, F. Microparafuso ortodôntico: instalação e orientação de higiene periimplantar. Rev. Clin. Ortodon. Dental Press, Maringá, v. 5, n. 1, p. 24-31, fev./mar. 2006.

14. NOJIMA, L. I. et al. Dispositivos temporários de ancoragem em Ortodontia. In: BERNARDES, J. Estética em Implantologia. 1. ed. São Paulo: Quintessence, 2006.
15. PITHON, M. M. Avaliação mecânica de mini-implantes ortodônticos. Dissertação (Mestrado)-Faculdade de Odontologia. Universidade Federal do Rio de Janeiro, Rio de Janeiro, 2008.

16. PARK, H. S.; KYUNG, H. M.; SUNG, J. H. A simple method of molar uprighting with micro-implant anchorage. J. Clin. Orthod., Boulder, v. 36, p. 592-596, 2002.

17. QUIRYNEM, M.; PAPAIOANNOU, W.; STEENBERGHE, D. $V$. The influence of titanium abutment surface roughness on plaque acumulation and gingivitis: short-term observations. Int. J. Oral Maxillofac. Implants, v. 11, no. 2, p. 169-178, 1996.

18. SUNG, J. H. et al. Microimplants in Orthodontics. Korea: Dentos Daegu, 2006.

19. SYKARAS, N. et al. Implant materials, designs and surface topographies: their effect on osseointegration. a literature review. Int. J. Oral Maxillofac. Implants, v. 15, no. 5, p. 675690, 2000

20. VILELLA, H.; BEZERRA, F.; LABOISSIÈRE, M. J. Microparafuso ortodôntico de titânio auto-perfurante (MPO): novo protocolo cirúrgico e atuais perspectivas clínicas. Innovations Implant Journal: Biomaterials and Esthetics, São Paulo, v. 1, n. 1, p. 46-53, maio 2006.

21. YANO, S. et al. Tapered orthodontic miniscrews induce bonescrew cohesion following immediate loading. Eur. J. Orthod., Oxford, v. 28, no. 6, p. 541-546, 2006.

22. WIECHMANN, D.; MEYER, U.; BÜCHTER, A. Success rate of mini and micro-implants used for orthodontic anchorage: a prospective clinical study. Clin. Oral Implants Res., Copenhagen, v. 18, p. 263-267, 2007.

\section{Endereço para correspondência}

Lincoln Issamu Nojima

Universidade do Brasil - UFRJ - Faculdade de Odontologia

Programa de Pós-graduação em Odontologia

Av. Brigadeiro Trompowsky, s/n $\mathrm{n}^{\circ}$ - Ilha do Fundão

CEP: 21.941-590 - Rio de Janeiro/RJ

E-mail: linojima@gmail.com 